News \& Views in ...

\title{
Biomarkers in Medicine
}

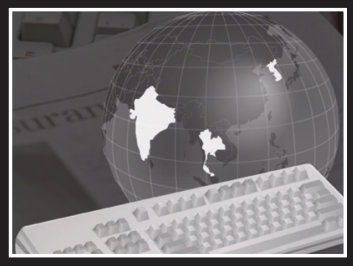

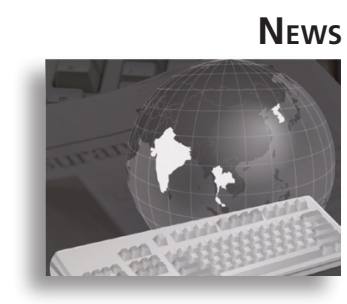

Research Highuights
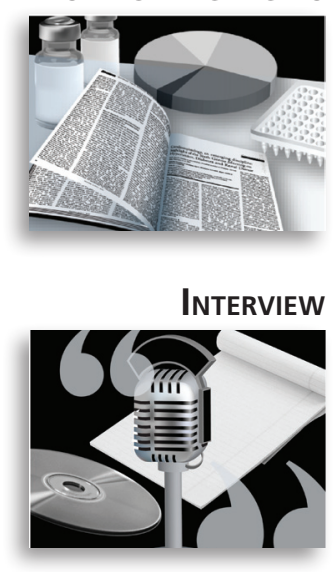

\section{Changes in circulating tumor cell levels found to be accurate in predicting castration-resistant prostate cancer treatment}

The results of a study investigating circulating tumor cells (CTCs) in prostate cancer have been presented at the European Association of Urology 13th Central European Meeting in Prague (Czech Republic). The study suggests that changes in CTC levels may be more accurate than PSA level changes in predicting outcomes for castration-resistant prostate cancer (CRPC).

The study involved collection of peripheral blood from CRPC patients following the fourth cycle of chemotherapy prior to docetaxel therapy. CTCs were detected

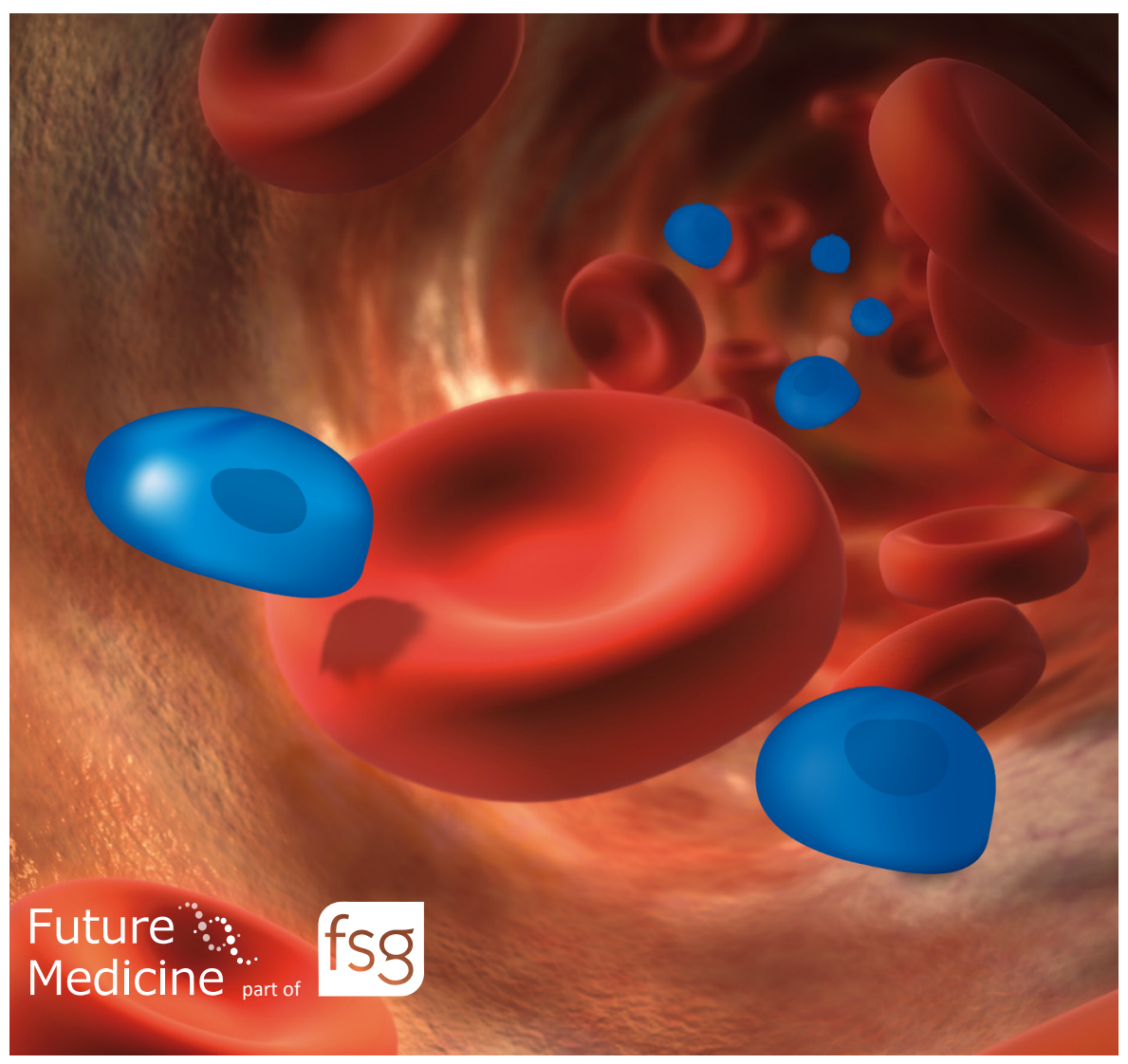

via immunomagnetic separation, followed by CTC cytolysis and multiplex PCR. The expression levels of PSA, PSMA and EGFR were subsequently quantified. The authors recorded serum PSA levels and respective antigen fragments before and during the course of chemotherapy, and subsequently compared the values. The correlation between the change in serum PSA and expression of CTC antigens was also evaluated during chemotherapy.

The study involved 26 patients with a median age of 72 years. Prior to chemotherapy, only two out of 26 patients were considered CTC negative, while during chemotherapy this increased to nine out of the 17 individuals for whom these values were available. Measured serum PSA level was observed to have the strongest correlation with the level of PSA gene fragment in CTCs. A favorable change in the number of CTCs occurred in over half of patients during chemotherapy; however, this change was not observed to correlate with the change in serum PSA level.

Explaining the aims and findings of the study, lead author Otakar Čapoun (University of Prague, Czech Republic) stated: "The research of the CTCs is of utmost importance, because nowadays there is no reliable marker of either cancer-specific or overall survival in CRPC patients. The goal of this study is to assess the possibility of the individualization of CRPC management. In cases with no favorable change in CTC detection during chemotherapy, the early switching to another therapy should be considered."

The researchers hope that the results of their study will be a step on the way 
towards CTC profiling being used to aid more accurate and tailored treatment selection for CRPC patients.
Source: Čapoun O et al. Prostate-specific antigen level and detection of circulating tumor cells in castration-resistant prostate cancer. Presented at: EAU 13th Central European Meeting. Prague, Czech Republic, 4-6 October 2013 (Abstract C163).

\section{Epigenetic biomarkers in the blood may identify early Parkinson's disease}

"Since early identification of pathological changes is crucial to enable therapeutic intervention in Parkinson's disease (PD) before major neurologic damage occurs, these findings could prove important in monitoring disease progression and the effectiveness of treatment," commented Paula Desplats (University of California, San Diego School of Medicine, CA, USA), lead author on the study, published in Epigenetics, which investigated genome-wide methylation in brain and blood samples of PD patients. The results provide evidence for an association between PD and DNA methylation that may be clinically utilized as a biomarker in the future.

This new research builds upon previous findings from University of California, San Diego in which a significant decrease in DNA methylation was identified in the frontal cortex of individuals affected by dementia with Lewy bodies and PD. In the current study, the researchers identified DNA methylation patterns in peripheral blood leukocytes

\section{Novel screening test for prostate} cancer yields positive results
Researchers from the University of Michigan (MI, USA) have recently developed a novel urine test that is more effective than the traditional screening test for prostate cancer.

The traditional blood test measures PSA to detect prostate cancer; however, despite the large number of men undergoing prostate biopsies, only approximately one-fifth of these cases will be diagnosed with cancer. In an effort to improve cancer screening, the Mi-Prostate Score (MiPS) is now being offered by the University of Michigan Health System. Researcher Scott Tomlins (University of Michigan) commented: "Many more men have elevated PSA than actually have cancer, but it can be difficult to determine this without biopsy. We need new tools to help patients and doctors make better decisions about what to do if serum PSA is elevated. MiPS helps with this."

MiPS was developed following the (occurring in $~ 50 \%$ of all prostate cancer cases) in which the position of two genes is altered and fusion of these genes identification of a genetic anomaly
$(T 2-E R G)$ occurs. Since gene fusion is highly indicative of cancer, the T2-ERG fusion along with a further marker, PCA3, is used to assess for cancer in the urine test, the effectiveness of which was validated in 2000 urine samples. MiPS incorporates this test with measurement of serum PSA and has been shown to be more accurate in predicting prostate cancer than PSA measurement alone.

Tomlins explained: "This combination test is not designed to say definitively at diagnosis whether a man has aggressive prostate cancer, but it can provide a more accurate estimate of the likelihood of having cancer and the likelihood of that cancer being aggressive." MiPS could therefore help patients and doctors make decisions about whether to undergo immediate biopsy.

\section{-Written by Alicia Gillard}

Source: University of Michigan Health System press release: www.cancer.med.umich. edu/news/early-detection-for-prostatecancer-2013.html and post-mortem frontal cortex samples from five PD patients compared with those of six age-matched, healthy controls using genome-wide DNA methylation profiles.

It was demonstrated that the methylation profiles gathered from the blood samples were able to distinguish those with PD from the healthy controls. The group identified 124 genes among both the blood and brain samples that could be utilized for further biomarker investigation.

$\mathrm{PD}$ is a neurodegenerative disorder for which diagnosis is not usually achieved until significant motor symptoms develop, by which point substantial damage to the midbrain (specifically the substantia nigra) has occurred. The evidence from the current study highlights a role for epigenetic alterations in the molecular mechanisms of neurodegeneration and furthermore suggests a future function for epigenetic markers in PD diagnosis.

\section{- Written by Elizabeth Webb}

Sources: Masliah E, Dumaop W, Galasko $D$, Desplats P. Distinctive patterns of DNA methylation associated with Parkinson disease: identification of concordant epigenetic changes in brain and peripheral blood leukocytes. Epigenetics 8(10), 1030-1038 (2013); University of California, San Diego Health Sciences News: http://ucsdhealthsciences. tumb/r.com / post/57717074992/ the-epigenetics-of-parkinsons-disease 


\section{ASCL1, a new biomarker for smokers' lung cancer?}

ASCL1, a protein involved in pulmonary neuroendocrine cell development and previously linked to regulation of thyroid and small-cell lung cancer development, as well as RET, an oncogene involved in the development of cancer, could be useful biomarkers for smoking-related lung adenocarcinoma.

George Vasmatzis and his colleagues at the Mayo Clinic (MN, USA) have recently published the results of a study on the use of these two biomarkers and their possible involvement in neuroendocrine differentiation in lung adenocarcinoma in Oncogene.

The authors analyzed the expression of ASCL1 in histological samples of various lung cancer types and they observed that the expression of this protein was present almost exclusively in smokers with lung adenocarcinoma. Moreover, when 367 microarray-based gene expression profiles in lung adenocarcinoma were analyzed, the RET oncogene expression was higher in ASCL1-positive than ASCL1negative samples; this expression was also correlated with a shorter overall survival.
In another experiment, Vasmatzis and colleagues blocked the expression of ASCL1 in adenocarcinoma cells: this silencing induced a reduction in the expression of $R E T$ and a slowing in tumor growth.

Vasmatzis pointed out that the particular relevance of the study stands in the fact that, according to the results, ASCL1 could become a potential target for therapy in the future, especially in smoking-related lung adenocarcinoma, which is particularly aggressive. Further studies are warranted to confirm the role of ASCL1 in the development of lung adenocarcinoma, but the results presented by Vasmatzis and colleagues indicate promising applications of ASCL1/RET targeting in future clinical trials.

- Written by Marco De Ambrogi

Source: Kosari F, Ida CM, Aubry MC et al. ASCL1 and RET expression defines a clinically relevant subgroup of lung adenocarcinoma characterized by neuroendocrine differentiation. Oncogene doi:10.1038/onc.2013.359 (2013) (Epub ahead of print).

\section{About the News}

The News highlights some of the most important events and research in the field of biomarkers. If you have newsworthy information, please contact:

Hannah Wilson, Commissioning Editor, Biomarkers in Medicine

Future Medicine Ltd, Unitec House, 2 Albert Place, London N3 1QB, UK

Tel.: +44 (0)20 8371 6090; hannah.wilson@futuremedicine.com 